\title{
Respuestas fisiológicas de Neotrypaea uncinata (Decapoda: Thalassinidea) a la hipoxia y al parasitismo por Ionella agassizi (Isopoda: Epicaridea)
}

Physiological responses of Neotrypaea uncinata (Decapoda: Thalassinidea) to hypoxia and parasitism by Ionella agassizi (Isopoda: Epicaridea)

\author{
Lorena P. Astete-Espinoza ${ }^{1}$, Claudia F. Garrido ${ }^{1}$ y Cristian W. Cáceres ${ }^{1}$
}

\author{
${ }^{1}$ Departamento de Ecología Costera, Facultad de Ciencias, Universidad Católica de la Santísima Concepción, Casilla 297, \\ Concepción, Chile. ccaceres@ucsc.cl
}

\begin{abstract}
Thalassinidean shrimps are common macrofaunal components of the intertidal and shallow subtidal zones of beaches and estuaries. These organisms present burrowing behavior and are exposed to low oxygen concentrations in their natural environment. In addition, the parasitic isopod lonella agassizi is frequently found in the branchial chamber of the ghost shrimp Neotrypaea uncinata. Previous studies have shown that the presence of this parasite affects the physiological condition of $\mathrm{N}$. uncinata, provoking a negative effect on reproduction. In this study we evaluated the effects of exposure to hypoxia and parasitism on host metabolic capacity. For this, we evaluated the effect of the parasite on area of gas exchange in the host and the ability to resist hypoxic conditions by evaluating haemolymphatic concentrations of proteins, haemocyanin, lactate, glucose and the activity of enzyme lactate-dehydrogenase (LDH). Our results indicate that the presence of the parasite reduces the gill area in N. uncinata. During exposition of the ghost shrimp to hypoxic conditions, this increases its hemolymphatic concentrations of glucose, lactate, and the activivity of LDH. These results are interpreted as the utilization of metabolic alternatives to aerobic metabolism during periods of oxygen deficiency.
\end{abstract}

Key words: Metabolism, haemolymph, ghost shrimp

\begin{abstract}
Resumen.- Los crustáceos Thalassinidae son componentes comunes de la macrofauna de los fondos blandos de playas y estuarios. Estos organismos son excavadores, encontrándose recurrentemente expuestos a bajas concentraciones de oxígeno en su ambiente natural. En adición, Neotrypaea uncinata se encuentra naturalmente parasitado en su cámara branquial por el isópodo bopírido lonella agassizi. En estudios previos se ha señalado que la presencia del parásito afecta la condición fisiológica de N. uncinata, provocándole un efecto negativo en la reproducción. En el presente trabaj o se estudian los efectos de la hipoxia y del parasitismo sobre la fisiología respiratoria de esta especie. Para esto, se midió el efecto del parásito sobre el área de intercambio gaseoso de N. uncinata y la capacidad de resistencia a condiciones hipóxicas a través de las mediciones de las concentraciones de metabólitos hemolinfáticos: proteínas, hemocianina, lactato, glucosa y la actividad de la enzima lactato-deshidrogenasa (LDH). Los resultados indican que por una parte la presencia del parásito disminuye significativamente el área de intercambio gaseoso en N. uncinata. Por otro lado, la exposición a hipoxia genera un aumento significativo de las concentraciones hemolinfáticas de hemocianina, glucosa, lactato, además de un aumento en la actividad de la enzima LDH. Estos resultados se interpretan como la utilización por parte de este crustáceo, de vías metabólicas alternativas a la aeróbica en periodos de déficit de oxígeno.
\end{abstract}

Palabras clave: Metabolismo, hemolinfa, camarón fantasma

\section{INTRODUCCION}

Los crustáceos excavadores habitan túneles y madrigueras en fondos blandos hipóxicos de playas y esteros, supliendo sus requerimientos de oxígeno y nutrientes a través de la remoción del sedimento (Dworschak et al. 2006). La singularidad de los ambientes en que habitan han hecho a estos organismos objeto de diversos trabajos de carácter fisiológico, los cuales han estado principalmente centrados en el estudio de las vías metabólicas utilizadas y en las respuestas respiratorias en condiciones de hipoxia aguda o crónica (Pritchard \& Eddy 1979, Lowery \& Tate 1986, Hill et al. 1991, Scholnick \& Snyder 1996, Astall et al. 1997). Dentro de los mecanismos compensatorios descritos se encuentran el aumento tanto de la concentración como de la afinidad con el oxígeno de la hemocianina. Además, se han descrito modificaciones del metabolismo de la glucosa y lactato, con un aumento de las concentraciones 
hemolinfáticas de lactato, el cual puede ser utilizado en la síntesis de glucosa (Albert \& Ellington 1985, Hagerman 1986, Hill et al. 1991, Anderson et al. 1994, Zou et al. 1996, Forster \& Graf 1995, Vismann \& Hagerman 1996, 2004, Olivera et al. 2004a, b, Maciel et al. 2008).

El camarón fantasma Neotrypaea uncinata (Milne Edwards 1837) es una especie de hábitos excavadores (Retamal 1975), que se encuentra parasitada en su cámara branquial desde etapas tempranas de su ciclo de vida por el isópodo Ionella agassizi (Bonnier 1900) (Muñoz \& George-Nascimento 1999). La presencia del parásito provoca el rompimiento de las branquias del hospedador (McDermontt 1991), con una probable reducción de la superficie de intercambio gaseoso, y funcionalmente genera una disminución de la disponibilidad de los recursos energéticos para el hospedador (Astete-Espinoza \& Cáceres 2000). Además se ha descrito que el parásito genera una reducción del crecimiento, afectando el desarrollo de los caracteres sexuales secundarios y la reproducción de su hospedador (Muñoz \& GeorgeNascimento 1999). Este sistema hospedador-parásito además se caracteriza por el gran tamaño del parásito en relación al hospedador (Astete-Espinoza \& Cáceres 2000), lo cual lo convierte en un caso especial de estudio acerca de los efectos del parásito, por su alta demanda nutricional, lo que influye directamente sobre la respuesta metabólica del hospedador.

La hipótesis central de este trabajo es que la presencia del parásito Ionella agassizi afecta los mecanismos respiratorios y las vías energéticas del nape Neotrypaea uncinata, limitando su capacidad para soportar periodos de déficit de oxígeno. Para evaluar esto se cuantificó el efecto destructivo del parásito sobre el área de intercambio gaseoso de las branquias, y se sometió a individuos parasitados y no parasitados a condiciones de hipoxia experimental, evaluando las variaciones tanto de las concentraciones hemolinfáticas de hemocianina, glucosa y lactato como de la actividad de la enzima lactato deshidrogenasa.

\section{Material y MÉtodos}

Entre octubre y noviembre de 1999, se recolectaron aproximadamente 300 ejemplares de Neotrypaea uncinata en la localidad costera de Lenga, Bahía San Vicente, zonacentro sur de Chile ( $\left.36^{\circ} 45^{\prime} \mathrm{S} ; 7^{\circ} 10^{\prime} \mathrm{W}\right)$, mediante una bomba extractora manual. De estos se seleccionaron 135 ejemplares de ambos sexos y de similar tamaño según apreciación visual. Posteriormente, cada ejemplar fue pesado $( \pm 0,01 \mathrm{~g})$, y medido en su longitud cefalotoráxica (LCT) $( \pm 0,01 \mathrm{~mm})$. La presencia del isópodo parásito Ionella agassizi fue evaluada mediante inspección visual de las cámaras branquiales. Antes de la experimentación, para evitar diferencias en el tamaño corporal, la LCT de los camarones fue comparada estadísticamente, mediante una ANDEVA, entre sexos y condición parasítica (con o sin isópodos), con el propósito de no tener un efecto de esta variable en las respuestas fisiológicas de los camarones.

Un grupo de camarones $(\mathrm{N}=55)$ fue utilizado para evaluar el efecto del parásito en el área superficial de las branquias del hospedador. Para esto, las branquias de cada individuo fueron disectadas bajo lupa binocular, contando el número de lamelas presentes en cada branquia. El área branquial fue estimada como el producto entre el ancho y largo de la lamela central multiplicado por el número lamelas presentes en cada cámara branquial (Torres et al. 1977).

El resto de los individuos capturados $(\mathrm{N}=80)$ fueron aclimatados en acuarios de laboratorio con agua de mar prefiltrada, acondicionado con sedimento de su lugar de origen, con aireación y temperatura constante $\left( \pm 14^{\circ} \mathrm{C}\right) \mathrm{y}$ fotoperíodo natural por tres días. Posteriormente, cuarenta individuos parasitados y cuarenta no parasitados de Neotrypaea uncinata fueron separados en cuatro tratamientos experimentales en igual proporción sexual, un control en normoxia y tres grupos sometidos a diferentes tiempos de exposición a hipoxia, 3,24 y $72 \mathrm{~h}(\mathrm{~N}=10$ por tratamiento). Todos los individuos fueron colocados en recipientes individuales con $400 \mathrm{~mL}$ de agua de mar filtrada $0,45 \mu \mathrm{m}$, con una salinidad de 33 ups y a una temperatura de $14 \pm 0,5^{\circ} \mathrm{C}$. Las condiciones de hipoxia se obtuvieron mediante burbujeo de nitrógeno (Cancino et al. 2003) hasta concentraciones de oxígeno menores a $1,0 \mathrm{mg} \mathrm{L}^{-1}$ para hipoxia, y de 4,5-5,0 $\mathrm{mg} \mathrm{L}^{-1}$ para normoxia; la concentración de oxígeno fue medida utilizando un oxigenómetro Hanna ${ }^{\circledR}$ HI 91410.

Al finalizar el tiempo de exposición a las condiciones de hipoxia, se extrajo de cada individuo, mediante punción de la región pericárdica del cefalotórax, $0,3 \mathrm{~mL}$ de hemolinfa, utilizando jeringas de $1 \mathrm{~mL}$ con $0,1 \mathrm{~mL}$ de solución EDTA (Sanders \& Childress 1990). Posteriormente las muestras fueron centrifugadas a 3000 rpm por 5 min y analizadas inmediatamente.

La respuesta metabólica a hipoxia fue evaluada mediante la determinación de los siguientes metabolitos hemolinfáticos: proteínas totales, glucosa, hemocianina, 
y lactato, además de la actividad de la enzima lactato deshidrogenasa (LDH).

La determinación de proteínas totales se realizó a través del método de Biuret (Layne 1957). La determinación de los niveles de hemocianina fue realizada según el método descrito por Chen \& Cheng (1993). Se utilizó el coeficiente de extinción de 17,26 E mM $1 \mathrm{~cm}^{-1}$ (Nickerson \& Van Holde 1971).

La determinación de los niveles de glucosa en la hemolinfa se realizó a través del método enzimático de punto final (Trinder), basado en la reacción glucosa oxidasa/peroxidasa. Para la determinación de los niveles de lactato, las muestras de hemolinfa fueron tratadas con TCA al $5 \%$ y centrifugadas a $3000 \mathrm{rpm}$ por $10 \mathrm{~min}$. La determinación se realizó por colorimetría utilizando la enzima lactato oxidasa (Sigma Chem. Co.) (Stillman \& Somero 1996). Los niveles de LDH fueron medidos utilizándose como sustrato Piruvato y NADH reducido. Para todas las determinaciones se utilizó un espectrofotómetro Turner UV-visible 690.

La relación entre área branquial y tamaño corporal de individuos parasitados y no parasitados se evaluó por medio de análisis de regresión lineal, las diferencias en pendientes e interceptos fueron evaluados mediante la metodología propuesta por Zar (1996).

Para evaluar el efecto del tiempo de exposición a condiciones de hipoxia y el efecto del parasitismo sobre los niveles de los metabolitos hemolinfáticos se realizó un análisis de la varianza (ANDEVA) de dos vías, con el tiempo y el parasitismo como factores fijos, seguido de la prueba a-posteriori de Tukey (Zar 1996). La homogeneidad de varianza se evaluó mediante la prueba de Levene, posteriormente los datos no homogéneos fueron transformados a logaritmo (Zar 1996). Los resultados se presentan como media y error estándar (X $\pm \mathrm{EE})$.

\section{Resultados}

Los ejemplares de Neotrypaea uncinata no presentan diferencias significativas en el tamaño corporal entre individuos parasitados y no parasitados o entre sexos, con una valor medio de $1,55 \pm 0,50 \mathrm{~g}\left(\right.$ ANDEVA $_{(1,131)}=0,24$ $P=0,63$ para sexo, $\mathrm{F}_{(1,131)}=3,49 P=0,07$ para parasitismo $\mathrm{y} \mathrm{F}_{(1,131)}=0,16 P=0,69$ para interacción entre parasitismo y sexo).

El análisis de la relación entre área superficial branquial y tamaño corporal indicó en ambos grupos una relación significativa con el tamaño corporal $\left(\mathrm{r}^{2}=0,25, P<0,05\right.$ para organismos parasitados y $\mathrm{r}^{2}=0,31, P<0,01$ para organismos no parasitados). La comparación de pendientes e interceptos indica que ambas pendientes son similares $\left(\mathrm{F}_{(1,51)}=0,001, P=0,98\right)$, pero que los interceptos son estadísticamente diferentes entre ambas regresiones $\left(\mathrm{F}_{(1,52)}=14,10, P<0,001\right)$ (Fig. 1).

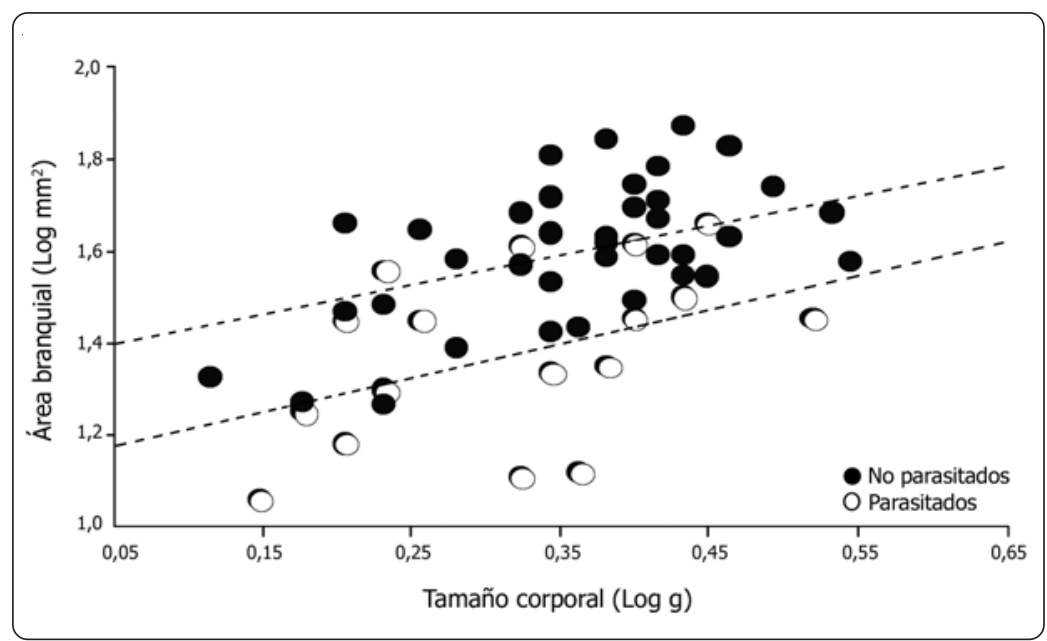

Figura 1. Relación entre tamaño corporal y área branquial en individuos parasitados y no parasitados de Neotrypaea uncinata $(y=1,128+0,821 x$ para individuos parasitados e $y=1,302+0,845 x$ para individuos no parasitados) / Relationship between body size and gill area in parasitized and non-parasitized individuals of Neotrypaea uncinata $(y=1.128+0.821 x$ for parasitized individuals and $y=1.302+0.845 x$ for non parasitized organisms) 


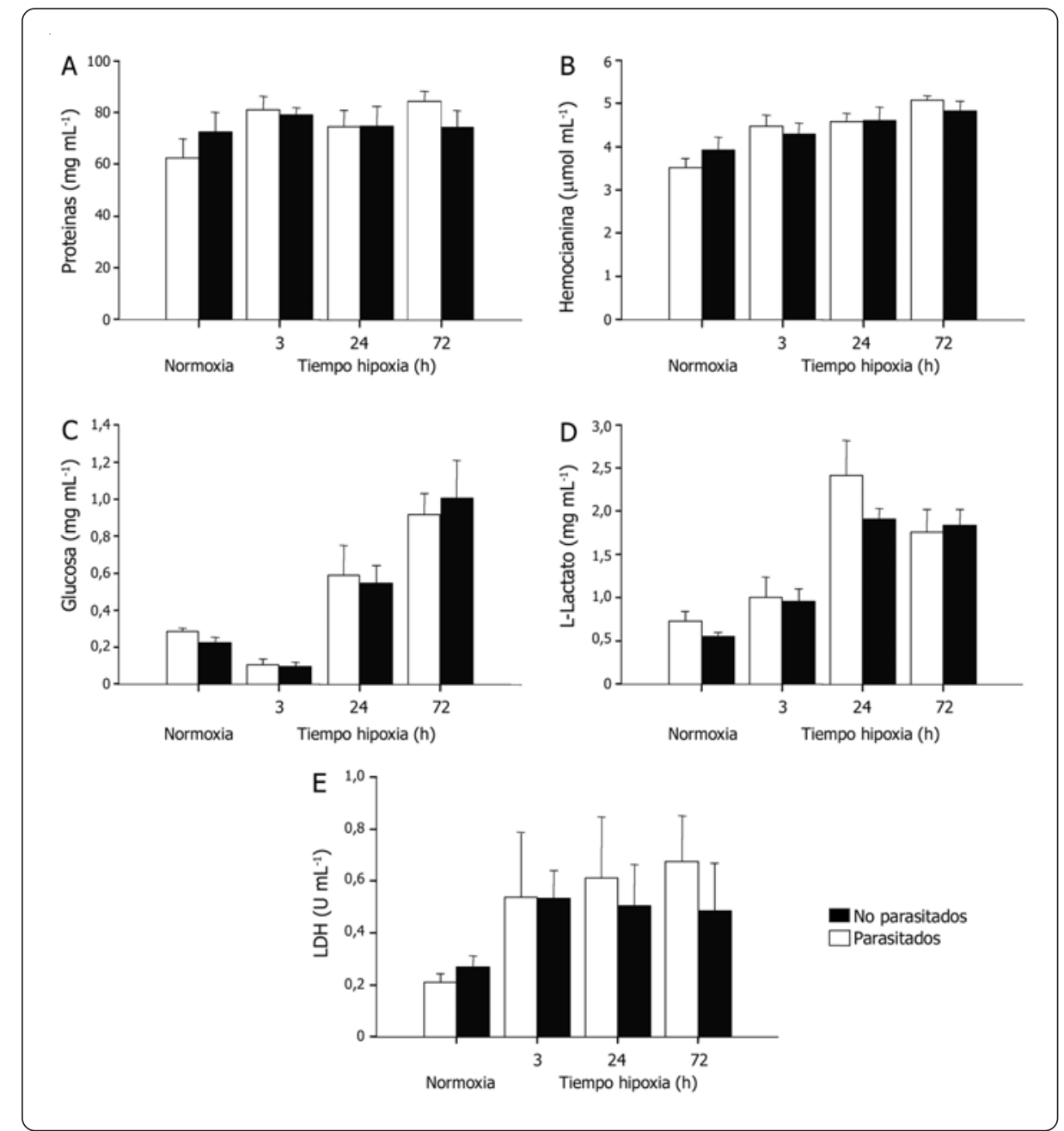

Figura 2. Concentraciones de metabolitos hemolinfáticos en individuos parasitados y no parasitados de Neotrypaea uncinata expuestos a normoxia y tres tiempos de exposición a hipoxia $(3,24$ y 72 h): A) Concentración de proteínas totales, B) Concentraciones de hemocianina, C) Concentraciones de glucosa, D) Concentraciones de lactato, E) Actividad LDH / Hemolymphatic concentrations in parasitized and non parasitized individuals of Neotrypaea uncinata exposed to normoxic and three times of hypoxia conditions (3,24 and 72 h): A) Total protein concentration, B) Hemocyanin concentrations, C) Glucose concentrations, D) Lactate concentrations, E) LDH activity
En las concentraciones de proteínas en la hemolinfa no se observó una influencia del tiempo de exposición a hipoxia $\left(\mathrm{F}_{(3,72)}=2,44 ; P=0,07\right)$, ni del parasitismo $\left(\mathrm{F}_{(1,72)}\right.$ $=0,03, P=0,87)$, ni de la interacción entre ambos factores $\left(\mathrm{F}_{(3,72)}=1,01 ; P=0,39\right)$. Con una media general de 75,18 $\pm 2,20 \mathrm{mg} \mathrm{mL}^{-1}$ (Fig. 2A) (Tabla 1).

En las concentraciones hemolinfáticas de hemocianina: se observó un efecto significativo del tiempo de exposición a hipoxia (ANDEVA, $\mathrm{F}_{(3,72)}=9,41, P=0,001$ ), siendo significativamente menor la concentración en normoxia en relación a los otros tratamientos experimentales $(3,72$ $\pm 0,18 \mu \mathrm{mol} \mathrm{mL}^{-1}$ ) (Tukey a-posteriori $P<0,05$ ) no observándose un efecto ni del parasitismo ni de la interacción entre ambos factores (ANDEVA $F_{(1,72)}=0,01$, $P=0,94$ para parasitismo y, $\mathrm{F}_{(3,72)}=0,77, P=0,52$ para interacción) (Fig. 2B).
Los niveles de glucosa en la hemolinfa fueron afectados significativamente por el tiempo de exposición a hipoxia (ANDEVA, $\mathrm{F}_{(3,72)}=51,87, P=0,001$ ) (Tabla 1), siendo diferentes las concentraciones medidas entre todos los tratamientos experimentales (Tukey a-posteriori $P<0,05$ ), el menor valor $\left(0,09 \pm 0,02 \mathrm{mg} \mathrm{mL}^{-1}\right)$ fue observado a las $3 \mathrm{~h}$ de exposición a hipoxia y el mayor valor a las $72 \mathrm{~h}$ de hipoxia $\left(0,96 \pm 0,11 \mathrm{mg} \mathrm{mL}^{-1}\right)$ (Fig. 2C). No se reportó un efecto significativo del parasitismo (ANDEVA, $\mathrm{F}_{(1,72)}=$ $0,90 ; P=0,34)$ o de la interacción entre hipoxia y parasitismo (ANDEVA, $\mathrm{F}_{(3,72)}=0,24 P=0,87$ ).

El análisis de las variaciones en las concentraciones de lactato presentes en la hemolinfa, indican que este parámetro fue significativamente influenciado por la hipoxia (ANDEVA $F_{(3,65)}=27,42 ; P=0,001$ ) (Tabla 1). La menor concentración se observó en los individuos 
Tabla 1. Resultado de ANDEVA para el efecto de parasitismo e hipoxia sobre las concentraciones hemolinfáticas en Neotrypaea uncinata; $g l=$ grados de libertad, MS=cuadrados medios; F= razón de $\mathbf{F}, \mathbf{P}=$ valor de probabilidad, *: indica diferencias significativas $(\mathbf{P}<0,05)$ / ANOVA results about the effects of hypoxia and parasitism over measured haemolymphatic concentrations in Neotrypaea uncinata; $\mathrm{gl}=$ degrees of freedom, $\mathrm{MS}=$ mean square, $\mathrm{F}=\mathrm{F}$ value, $\mathrm{P}=$ probability value, *: indicates significant differences $(P<0.05)$

\begin{tabular}{|c|c|c|c|c|c|}
\hline Variable de respuesta & Efecto & GL & MS & $\mathrm{F}$ & Probabilidac \\
\hline \multirow[t]{4}{*}{ Proteínas } & Hipoxia & 3 & 0,037 & 2,44 & 0,07 \\
\hline & Parasitismo & 1 & 0,01 & 0,03 & 0,87 \\
\hline & Interacción & 3 & 0,02 & 1,01 & 0,39 \\
\hline & Error & 72 & 0,02 & & \\
\hline \multirow[t]{4}{*}{ Hemocianina } & Hipoxia & 3 & 0,06 & 9,41 & $<0,01^{*}$ \\
\hline & Parasitismo & 1 & 0,01 & 0,01 & 0,94 \\
\hline & Interacción & 3 & 0,01 & 0,77 & 0,52 \\
\hline & Error & 72 & 0,01 & & \\
\hline \multirow[t]{4}{*}{ Glucosa } & Hipoxia & 3 & 4,70 & 51,57 & $<0,01 *$ \\
\hline & Parasitismo & 1 & 0,08 & 0,90 & 0,34 \\
\hline & Interacción & 3 & 0,02 & 0,24 & 0,87 \\
\hline & Error & 72 & 0,09 & & \\
\hline \multirow[t]{4}{*}{ Lactato } & Hipoxia & 3 & 1,22 & 27,42 & $<0,01 *$ \\
\hline & Parasitismo & 1 & 0,01 & 0,06 & 0,80 \\
\hline & Interacción & 3 & 0,01 & 0,25 & 0,86 \\
\hline & Error & 65 & 0,04 & & \\
\hline \multirow[t]{4}{*}{ LDH } & Hipoxia & 3 & 0,50 & 1,81 & 0,15 \\
\hline & Parasitismo & 1 & 0,05 & 0,18 & 0,67 \\
\hline & Interacción & 3 & 0,60 & 2,17 & 0,10 \\
\hline & Error & 72 & 0,27 & & \\
\hline
\end{tabular}

mantenidos en condiciones de normoxia y fue de $0,64 \pm$ $0,06 \mathrm{mg} \mathrm{mL}^{-1}$, mientras que la mayor concentración fue detectada en los individuos sometidos a $24 \mathrm{~h}$ de hipoxia alcanzando un valor de $2,16 \pm 0,21 \mathrm{mg} \mathrm{mL}^{-1}$, el análisis $a$ posteriori indica que los valores de lactato detectados a las 24 y $72 \mathrm{~h}$ de hipoxia son significativamente mayores que los observados en condiciones de normoxia y $3 \mathrm{~h}$ de exposición a hipoxia (Tukey a-posteriori $P<0,05$ ) (Fig. 2D). El efecto tanto del parasitismo como de la interacción no fue estadísticamente significativo (ANDEVA $\mathrm{F}_{(1,62)}=$ $0,06, P=0,80 ; \mathrm{F}_{(3,65)}=0,25, P=0,86$, para parasitismo e interacción respectivamente).

Finalmente las actividades de la enzima LDH medidas en la hemolinfa no experimentaron una influencia del tiempo de exposición a hipoxia (ANDEVA, $\mathrm{F}_{(3,72)}=1,85$, $P=0,15$ ), ni del parasitismo (ANDEVA, $\mathrm{F}_{(1,72)}=0,18, P=$ $0,67)$ ni de la interacción entre ambos factores hipoxia
(ANDEVA, $\mathrm{F}_{(3,72)}=2,17, P=0,10$ ) (Tabla 1). El valor promedio de actividad de la $\mathrm{LDH}$ fue de $0,47 \pm 0,06 \mathrm{U}$ $\mathrm{mL}^{-1}$ (Fig. 2E).

\section{Discusión}

El metabolismo aeróbico es mas eficiente que le metabolismo anaeróbico (Strayer 1995) y clave en la evolución de los organismos pluricelulares (Hickman et al. 2001). Por esto, el estudio de los mecanismos que les permitirían sobrevivir en ambientes con poco oxígeno, es relevante no solo desde la perspectiva de la fisiología, sino que también desde el punto de vista tanto de la ecología como de la evolución de estos organismos.

La Bahía San Vicente, se caracteriza por constantes fluctuaciones de salinidad, temperatura y oxígeno en la columna de agua y por presentar sedimentos con bajo 
potencial redox, característico de ambientes hipóxicos (Stuardo et al. 1993, Siemens et al. 2001, Rudolph et. al. 2002). Estas fluctuaciones condicionan la fisiología de los camarones fantasmas que habitan en esta bahía, por lo que deben modificar regularmente su metabolismo para adecuarse a las características del medio (Anderson 1977, Pritchard \& Eddy 1979, Anderson et al. 1991, 1994, Forster \& Graf 1995). En el caso particular de Neotrypaea uncinata, su habitat se ha descrito como altamente variable en los parámetros mencionadas (Stuardo et al. 1993, Siemens et al. 2001). Además de estas condiciones ambientales, se suma el parasitismo por Ionella agassizi, que a nivel individual afecta la reproducción de su hospedador, lo cual puede tener efectos a nivel poblacional y comunitario (Mouritsen \& Poulin 2002).

La presencia de Ionella agassizi en la cámara branquial del camarón fantasma Neotrypaea uncinata afecta la superficie de intercambio gaseoso, presentando los individuos parasitados una disminución significativa del área branquial en comparación con los organismos no parasitados (Fig. 1). Esta disminución se produce probablemente por el gran tamaño de isópodo y el efecto del roce sobre las branquias del hospedador (Anderson \& Dale 1981, McDermott 1991). Dado que la superficie de intercambio es clave en los procesos difusivos de intercambio de gases, esta disminución del área branquial implicaría que en iguales condiciones de requerimientos energéticos, los individuos parasitados dispondrían de una menor cantidad de oxígeno para suplir sus demandas, pudiendo generar incluso en condiciones normales de oxígeno un estado de hipoxia funcional, comprometiendo así la viabilidad de los organismos parasitados (AsteteEspinoza \& Cáceres 2000). No obstante lo anterior, no se observaron mecanismos compensatorios durante su desarrollo en los napes parasitados, ya que las pendientes para la relación área branquial-tamaño del hospedador, son similares tanto para individuos con parásitos como individuos no parasitados (Fig. 1)

Se ha descrito que en condiciones de falta de oxígeno los crustáceos favorecen la síntesis de hemocianina, que representa la mayor parte de las proteínas hemolinfáticas, para compensar la baja en la disponibilidad de oxígeno (Hagerman 1983, 1986, Hagerman \& Baden 1988, Baden et al. 1990, 1994, Scholnick \& Snyder 1996, Mangum 1997). Esto concuerda con lo encontrado en este estudio, donde no se encontraron diferencias en las concentraciones de proteínas en la hemolinfa, pero si diferencias en la concentración de hemocianina en función del tiempo de exposición a hipoxia (Fig. 2B), lo cual sugiere que las otras proteínas de la hemolinfa están siendo utilizadas como sustrato para la síntesis del pigmento. En normoxia se observan niveles similares de hemocianina, lo que concuerda con lo visto en punto anterior en el sentido que a pesar del gran tamaño del parásito y su gran efecto en la superficie de intercambio, este no genera un estado de hipoxia funcional en napes parasitados.

Por otra parte, el lactato es el principal producto final de la hipoxia en crustáceos, tiende a disminuir luego de un período prolongado bajo esta condición (Hagerman \& Vismann 1995, Zou et al. 1996). Los organismos que habitan en ambientes hipóxicos utilizan vías metabólicas anaeróbicas para la obtención de energía, mecanismo que requiere de individuos altamente tolerantes al lactato. De este modo el lactato es usado como sustrato gluconeogénico, obteniéndose un aumento en las concentraciones de glucosa circulante, e incluso en algunos casos un aumento del glicógeno en el hepatopancreas (Hagerman \& Szaniawska 1988, 1990, Hill et al. 1991, Anderson et al. 1994, De Wachter et al. 1997, Oliveira et al. 2004a, b). Lo anterior respalda los resultados obtenidos para $N$. uncinata en este estudio, ya que a las $3 \mathrm{~h}$ de exposición a hipoxia las concentraciones de lactato aumentan alcanzando niveles máximos a las $24 \mathrm{~h}$ (Fig. 2D), disminuyendo en las horas posteriores. Estos resultados se corroboran con las variaciones obtenidas en los niveles de la enzima lactato deshidrogenasa (LDH), encargada de la reacción de transformación de piruvato a lactato y de lactato a piruvato; éstos aumentaron en el tiempo de manera similar a las concentraciones de lactato en la hemolinfa, aunque debido a la alta variabilidad, las diferencias no fueron significativas (Fig. 2E).

Al relacionar los niveles de glucosa con los de lactato, se observó que se incrementan luego de $24 \mathrm{~h}$ de hipoxia manteniéndose hacia las $72 \mathrm{~h}$, con un valor promedio aproximadamente cuatro veces mayor con respecto a los individuos sometidos a normoxia; reduciéndose las concentraciones de lactato a través del tiempo de exposición. Los mayores niveles de glucosa encontrados en individuos en condiciones de normalidad, con respecto a los ejemplares sometidos a $3 \mathrm{~h}$ de hipoxia, corresponderían a la respuesta adaptativa a esta nueva condición lo que involucra un mayor costo energético por parte de $N$. uncinata y por ende una rápida utilización de la glucosa circulante (Bridges \& Brand 1980, Zou et al. 1996, De Wachter et al. 1997).

Los crustáceos bajo condiciones de estrés, liberan la hormona hiperglicemiante elevando inmediatamente los niveles de glucosa circulante por un período corto de 
tiempo (Santos \& Keller 1993, Santos et al. 1997). Este tipo de respuesta corresponde a una medida compensatoria frente a la elevada actividad energética que genera el estrés en un organismo, especialmente en crustáceos. No obstante, esta condición puede ser observada en Neotrypaea uncinata a las $24 \mathrm{~h}$ donde se evidencia un incremento significativo de la concentración de glucosa circulante.

Los niveles de lactato encontrados en Neotrypaea uncinata, correlacionados con las concentraciones de glucosa estarían indicando que estos individuos utilizarían alternativamente vías anaerobias ya que debido a su conducta excavadora en un medio de concentraciones de oxígeno variables. Contrario a lo esperado, esta respuesta adaptativa no estaría más acentuada en los individuos parasitados, debido a un efecto compensatorio a nivel molecular o fisiológico que le permitiría al camarón fantasma contrarrestar el efecto del parasitismo o el parásito genere bajas demandas a su hospedador.

\section{Agradecimientos}

Los autores agradecen de manera especial a Ximena Urrutia, Marcelina Peñaloza y Pablo Quijada por su colaboración en los análisis bioquímicos. A los revisores anónimos por sus comentarios que mejoraron sustantivamente el trabajo. Se agradece a Don José Escobar por su ayuda en la captura de especímenes. Financió Proyecto Fondecyt 1980442.

\section{LITERATURA CITADA}

Albert JL \& WR Ellington. 1985. Patterns of metabolism in the stone crab, Menippe mercenaria, during severe hypoxia and subsequent recovery. Journal of Experimental Zoology 234: $175-183$.

Anderson G. 1977. The effects of parasitism on energy flow through laboratory shrimp populations. Marine Biology 42: 229-251.

Anderson G \& WE Dale. 1981. Probopyrus pandalicola (Packard) (Isopoda, Epicaridea): morphology and development of larvae culture. Crustaceana 41: 143-161.

Anderson SJ, RJAAtkinson \& AC Taylor. 1991. Behavioural and respiratory adaptations of the mud-burrowing shrimp Calocaris macandreae Bell (Thalassinidea: Crustacea) to the burrow environment. Ophelia 134: 143-156.

Anderson SJ, AC Taylor \& RJA Atkinson. 1994. Anaerobic metabolic during anoxia in the burrowing shrimp Calocaris macandreae Bell (Crustacea: Thalassinidea). Comparative Biochemistry and Physiology A 108: 515-522.
Astall CA, AC Taylor \& RJA Atkinson. 1997. Behavioural and physiological implications of a burrow-dwelling lifestyle for two species of upogebiid mud-shrimp (Crustacea: Thalassinidea). Estuarine, Coastal and Shelf Science 44: 155-168.

Astete-Espinoza L \& CW Cáceres. 2000. Efecto del parasitismo del isópodo bopírido Ionella agassizi (Isopoda: Epicaridea) (Bornier, 1900) sobre la fisiología respiratoria del nape Neotrypaea uncinata (M. Edwards, 1837) (Decapoda: Thalassinidea). Revista Chilena de Historia Natural 73: 243-252.

Baden PS, L Pihl \& R Rosenberg. 1990. Effects of oxygen depletion on the ecology, blood physiology and fishery of the Norway lobster Nephrops norvegicus. Marine Ecology Progress Series 67: 141-155.

Baden PS, MH Depledge \& L Hagerman. 1994. Glycogen depletion and altered copper and manganese handling in Nephrops norvegicus following starvation and exposure to hypoxia. Marine Ecology Progress Series 103: 65-72.

Bridges CR \& AR Brand. 1980. The effect of hypoxia on oxygen consumption and blood lactate levels of some marine crustacea. Comparative Biochemistry and Physiology A 65: 399-409.

Cancino JM, JA Gallardo \& FA Torres. 2003. Combined effects of dissolved oxygen concentration and water temperature on embryonic development and larval shell secretion in the marine snail Chorus giganteus (Gastropoda: Muricidae). Marine Biology 142: 133-139.

Chen JC \& SY Cheng. 1993. Hemolymph, PCO2, hemocyanin, protein levels and urea excretions of Penaeus monodon exposed to ambient ammonia. Aquatic Toxicology 27: 281-292.

De Wachter B, FJ Sartoris \& HO Pörtner. 1997. The anaerobic end product lactate has a behavioural and metabolic signaling function in the shore crab Carcinus maenas. Journal of Experimental Biology 200: 1015-1024.

Dworschak PC, H Koller \& D Abed-Navandi. 2006. Burrow structure, burrowing and feeding behaviour of Corallianassa longiventris and Pestarella tyrrhen (Crustacea, Thalassinidea, Callianassidae). Marine Biology 148: 1369-1382.

Forster S \& S Graf. 1995. Impact of irrigation on oxygen flux into sediment: intermittent pumping by Callianassa subterranea and "piston-pumping" by Lanice conchilega. Marine Biology 123: 335-346.

Hagerman L. 1983. Haemocyanin concentration of juvenile lobsters (Homarus gammarus) in relation to moulting cycle and feeding. Marine Biology 77: 11-17.

Hagerman L. 1986. Haemocyanin concentration in the shrimp Crangon crangon (L.) after exposure to moderate hypoxia. Comparative Biochemistry and Physiology A 85: 721-724. 
Hagerman L \& SP Baden. 1988. Nephrops norvegigus: Field study of effects of oxygen deficiency on haemocyanin concentration. Journal Experimental Marine Biology and Ecology 116: 135-142.

Hagerman L \& A Szaniawska. 1988. Respiration, ventilation and circulation under hypoxia in the glacial relict Saduria (Mesidotea) entomon. Marine Ecology Progress Series 47: 55-63.

Hagerman L \& A Szaniawska. 1990. Anaerobic metabolism strategy of the glacial relict isopod Saduria (Mesidotea) entomon. Marine Ecology Progress Series 59: 91-96.

Hagerman L \& B Vismann. 1995. Anaerobic metabolism in the shrimp Crangon crangon exposed to hypoxia, anoxia and hydrogen sulfide. Marine Ecology Progress Series 123: 235-240.

Hickman CP Jr, LS Roberts \& A Larson. 2001. Integrated principles of zoology, 918 pp. McGraw Hill, Boston.

Hill AD, AC Taylor \& RHC Strang. 1991. Physiological and metabolic response of the shore crab Carcinus maenas (L) during environmental anoxia and subsequent recovery. Journal Experimental Marine Biology and Ecology 150: 31-50.

Layne E. 1957. Spectrographic and turbidimetric methods for measuring proteins. En: Kolowich SP \& NO Kaplan (eds). Methods in enzymology, pp. 447-454. Academic Press, New York.

Lowery TA \& LG Tate. 1986. Effect of hypoxia on hemolymph lactate and behavior of the blue crab Callinectes sapidus (Rathbun) in the laboratory and field. Comparative Biochemistry and Physiology A 85: 689-692.

Maciel JE, F Souza, S Valle, LC Kucharsky \& RS Martins Da Silva. 2008. Lactate metabolism in the muscle of the crab Chasmagnathus granulatus during hypoxia and posthypoxia recovery. Comparative Biochemistry and Physiology A 151: 61-65.

Mangum CP. 1997. Adaptation of the oxigen transport system to hypoxia in the blue crab, Callinectes sapidus. American Zoologist 37: 604-611.

McDermott JJ. 1991. Incidence and host-parasite relationship of infection (Crustacea, Isopoda, Bopyridae) in the Brachyuran crab Pachygrapsus transversus from Bermuda. Ophelia 33: 71-95.

Mouritsen KN \& R Poulin. 2002. Parasitism, community structure and biodiversity in intertidal ecosystems. Parasitology 124: 8101-8117.

Muñoz G \& M George-Nascimento. 1999. Efectos reproductivos en la simbiosis entre camarones fantasmas (Decapoda: Thalassinidea) e isópodos bopíridos (Isopoda: Epicaridea). Revista Chilena de Historia Natural 72: 4958.
Nickerson KW \& KE Van Holde. 1971. A comparison of molluscan and arthropod hemocyanin. I. Circular dichroism and absorption spectrum. Comparative Biochemistry and Physiology B 39: 855-872.

Oliveira GT, P Eichler, ICR Rossi \& RSM Da Silva. 2004a. Hepatopancreas gluconeogenesis during anoxia and postanoxia recovery in Chasmagnathus granulata crabs maintained on high-protein or carbohydrate-rich diets. Journal of Experimental Zoology 301A: 240 -248.

Oliveira GT, ICR Rossi, LC Kucharski \& RSM Da Silva. 2004b. Hepatopancreas gluconeogenesis and glycogen content during fasting in crabs previously maintained on a high-protein or carbohydrate-rich diet. Comparative Biochemistry and Physiology A 137: 383-390.

Pritchard AW \& S Eddy. 1979. Lactate formation in Callianassa californiensis and Upogebia pugettensis. Marine Biology 50: 249-253.

Retamal MA. 1981. Catálogo ilustrado de los crustáceos decápodos de Chile. Gayana Zoología 44: 7-110.

Rudolph A, R Ahumada \& C Pérez. 2002. Dissolved oxygen content as an index of water quality in San Vicente Bay, Chile $\left(36^{\circ} \mathrm{S}\right)$. Environmental Monitoring and Assessment 68: 89-100.

Sanders NK \& JJ Childress. 1990. Adaptations to the deepsea oxygen minimun layer: Oxygen binding by the hemocyanin of the bathypelagic Mysid, Gnathophausia ingens Dohrn. The Biological Bulletin 178: 286-294.

Santos AE \& R Keller. 1993. Crustacean hyperglycemic hormone $(\mathrm{CHH})$ and the regulation of carbohidrate metabolism: current perspectives. Comparative Biochemistry and Physiology A 106: 405-411.

Santos EA, ME Nery, R Keller \& AA Gonçalves. 1997. Evidence for the involvement of the crustacean hyperglycemic hormone in the regulation of lipid metabolism. Physiological Zoology 70: 415-420.

Scholnick DA \& GK Snyder. 1996. Response of the tadpole shrimp Triops longicaudatus to hypoxia. Crustaceana 69: 937-948.

Siemens RA, SM Stephen \& JM Cancino. 2001. The effect of physical and chemical parameters on the macroinfaunal community structure of San Vicente bay, Chile. Revista Chilena de Historia Natural 74(2): 429-444.

Stillman HS \& GN Somero. 1996. Adaptation to temperature stress and aerial exposure in congeneric species of intertidal porcelain crabs (genus Petrolisthes): correlation of physiology, biochemistry and morphology with vertical distribution. The Journal of Experimental Biology 199: 1845-1855.

Strayer L. 1995. Biochemistry, 1089 pp. W.H. Freeman and Company, New York. 
Stuardo J, C Valdovinos, R Figueroa \& A Ochipinti. 1993.

Los ambientes costeros del Golfo de Arauco y área adyacente. Serie Monografías Científicas, Centro EULA, Concepción, Chile 9: 1-157.

Torres JJ, DJ Gluck \& JJ Childress. 1977. Activity and physiological significance of the pleopods in the respiration of Callianassa californiensis (Dana) (Crustacea: Thalassinidea). The Biological Bulletin 152: 134-146.

Vismann B \& L Hagerman. 1996. Recovery from hypoxia with and without sulphide in Saduria entomon: oxygen debt reduced sulphur and anaerobic metabolites. Marine Ecology Progress Series 143: 131-139.

Zar JH. 1996. Biostatistical analysis, 718 pp. Prentice-Hall, Englewood Cliffs.

Zou E, N Du \& W Lai. 1996. The effect of severe hypoxia on lactate and glucose concentration in the blood of the Chinese freshwater crab Eriocheir sinensis (Crustacea: Decapoda). Comparative Biochemistry and Physiology A 114: 105-109.

Recibido el 3 de diciembre de 2009 y aceptado el 19 de agosto de 2010 